\title{
Comparison between Plasma and Urine Thiocyanates and Urinary Cotinine Determinations as Indicators of Cigarette Smoking
}

\author{
Haj Mouhamed Dhouha, ${ }^{*}$ Ezzaher Asma, Neffati Fadoua, Douki Wahiba, \\ and Najjar Mohamed Fadhel
}

Laboratory of Biochemistry-Toxicology, University Hospital of Monastir, Avenue 1er Juin, 5000 Monastir, Tunisia

(Received May 7, 2009; Accepted August 23, 2009)

\begin{abstract}
The aim of our study was to demonstrate the suitability of urinary cotinine and plasma thiocyanates $\left(\mathrm{SCN}^{-}\right)$as indicators of tobacco smoking and to investigate the correlation among urinary cotinine, plasma and urine $\mathrm{SCN}^{-}$ and number of cigarettes smoked per day and smoking topography. The initial study was conducted with 256 individuals: 143 nonsmokers aged $44.20 \pm 15.81$ years and 113 current smokers aged $38.02 \pm 17.49$ years. Subjects were classified into smokers or nonsmokers based on a questionnaire. Cotinine levels were measured using the enzymatic colorimetric method and $\mathrm{SCN}^{-}$using selective electrodes. Urinary cotinine and plasma $\mathrm{SCN}^{-}$levels are both significantly higher in smokers than in nonsmokers and correlate well with the number of cigarettes smoked per day. Urinary cotinine was significantly correlated with duration of consumption $\left(\mathrm{F}_{3-109}=3.43 ; p=0.019\right.$; $r=0.9961)$, and there was a negative correlation between body mass index and urinary cotinine $(r=0.9989$, $p<0.05)$. Urinary cotinine and plasma $\mathrm{SCN}^{-}$levels discriminate between smokers and nonsmokers and increase when smoking exceeds 20 cigarettes/day and duration of consumption exceeds 5 years.
\end{abstract}

Key words — cigarette smoking, cotinine, thiocyanate

\section{INTRODUCTION}

Self-reported data on smoking status, usually obtained by interview or questionnaire, are commonly used to measure the effectiveness of intensive smoking cessation programs or the relationships of smoking to the risk of certain diseases. The reliability of such data is limited: the individual may underreport in response to cessation program pressures or underestimate the extent of his/her smoking level. Programs may thus be overestimated, and the association of smoking habit changes with disease endpoints may be underestimated.

Tobacco exposure can be assessed by the measurement of several markers in biological fluids. These markers are more or less specific for tobacco, and the different methods to measure them differ in terms of sensitivity and specificity. The clinician prescribing a dosage for a patient must take all these parameters into account to make an accurate

\footnotetext{
*To whom correspondence should be addressed: Laboratory of Biochemistry-Toxicology, University Hospital of Monastir, Avenue 1er Juin, 5000 Monastir, Tunisia. Tel.: +21-673-461144; Fax: +21-673-460-678; E-mail: hajdhouha@yahoo.fr
}

choice. Tobacco biochemical markers have been demonstrated to provide reliable verification of patients' smoking status, with cotinine being the analyte of choice for quantifying nicotine intake during tobacco consumption ${ }^{1)}$ and exposure to environmental tobacco smoke (ETS).

Cotinine is the major proximate metabolite of nicotine and has been widely used as a biomarker of ETS exposure. Cotinine levels in the plasma, urine, and saliva of nonsmokers have been used in the assessment of ETS exposure and risk of ETS-related lung cancer. Another biomarker, thiocyanates $\left(\mathrm{SCN}^{-}\right)$, has been used as a biomarker for ETS exposure; although it displays a lack of specificity and sensitivity, $\mathrm{SCN}^{-}$, a metabolite of hydrogen cyanide gas consumed while smoking, has been proposed as a biochemical index of smoking exposure and risk. ${ }^{2)}$

In the present study, we analyzed the usefulness of the main biological tobacco markers (cotinine and $\mathrm{SCN}^{-}$) in discriminating active smokers from nonsmokers and determined the correlation between these biological tobacco markers and number of cigarettes smoked per day and smoking topography (puffing pattern of a smoker including

(C)2009 The Pharmaceutical Society of Japan 
puff volume, puff duration, puff profile, puff frequency, puff interval, and puff number).

\section{MATERIALS AND METHODS}

Population — The initial study was conducted in 256 individuals: 143 were nonsmokers aged 44.20 \pm 15.81 years and 113 current smokers aged $38.02 \pm$ 17.49 years. "Nonsmokers" are defined as those replying "no" to the questions "have you ever smoked cigarettes on a daily basis?" and "have you smoked a cigarette in the past week?" Another question, "do you now smoke cigarettes on a daily basis?" separated exsmokers from smokers.

Samples — Urine samples were obtained from the volunteers, including adult males and females who were smokers and nonsmokers. These samples were either used the same day or frozen at $-20^{\circ} \mathrm{C}$ until required for analysis. All the samples were analysed for cotinine and $\mathrm{SCN}^{-}$. Blood samples were drawn from an antecubital vein into Vacutainers containing EDTA (Becton Dickinson, Franklin Lakes, NJ, U.S.A.). After centrifugation, aliquots of plasma were frozen at $-20^{\circ} \mathrm{C}$ until analyzed for $\mathrm{SCN}^{-}$.

Smoking Questionnaire —— The smoking questionnaire combined both interviewerand selfadministered items; only interviewer-administered items are analyzed here. All patients were questioned about their medical history; sociodemographic characteristics included age, gender, education, and employment. Routine medical examination included measurements of weight $(\mathrm{W})$ and height $(\mathrm{H})$. Body mass index (BMI) was calculated as $\mathrm{W}$ divided by $\mathrm{H}$ squared $\left[\mathrm{BMI}=\mathrm{W} / \mathrm{H}^{2}\left(\mathrm{~kg} / \mathrm{m}^{2}\right)\right]$.
Laboratory Analysis — Cotinine levels were determined using the enzymatic colorimetric method (Konélab 30 $0^{\mathrm{TM}}$, Thermo Electron Corporation, Ruukintie, Finland) and expressed as micrograms per micromol of creatinine in urine. Plasma and urine $\mathrm{SCN}^{-}$levels were determined using selective electrodes (Ionometer Seven Multi S80, Mettler Toledo, Schwerzenbach, Switzerland) and expressed as milligrams per gram of creatinine in urine and milligrams per liter in serum.

Statistical Analysis — All results are presented as mean \pm S.D. Statistical analysis was performed using Student's $t$-test. Standard descriptive statistics, correlation coefficients, and significance tests were calculated using the Statistical Analysis System for examining the relationship between biochemical markers and cigarettes smoked per day because of the nonnormal distribution of the final puff. Differences between groups were evaluated with analysis of variance (ANOVA), followed by the Tukey post-hoc tests. A $p$ value of less than 0.05 was considered to represent a statistically significant difference between groups.

\section{RESULTS}

Table 1 summarizes the average levels of urinary and plasma $\mathrm{SCN}^{-}$and urinary cotinine, reported smoking status, gender, and alcoholic beverage consumption. We found a significant dissimilarity between smokers and nonsmokers in the urinary cotinine $\left(p<10^{-7}\right)$ and plasma $\mathrm{SCN}^{-}$levels $(p<0.0005)$ but not in urinary $\mathrm{SCN}^{-}$levels.

As shown in Table 1, there were some relevant gender differences. The urinary cotinine and plasma

Table 1. Variation of Urinary Cotinine, Urinary and Plasma SCN ${ }^{-}$Levels According Smoking Status, Gender and Alcoholic Beverage

\begin{tabular}{|c|c|c|c|c|c|c|c|}
\hline \multicolumn{2}{|c|}{ Parameters } & \multirow{2}{*}{$\begin{array}{c}\text { Urinary } \\
\text { cotinine } \\
(\mu \mathrm{g} / \mu \mathrm{mol} \mathrm{Cr})\end{array}$} & \multirow{3}{*}{$p$} & \multirow{2}{*}{$\begin{array}{c}\text { Urinary } \\
\mathrm{SCN}^{-} \\
(\mathrm{mg} / \mathrm{g} \mathrm{Cr})\end{array}$} & \multirow{2}{*}{\multicolumn{2}{|c|}{$\begin{array}{c}\text { Plasma } \\
\mathrm{SCN}^{-} \\
(\mu \mathrm{mol} / \mathrm{l})\end{array}$}} & \multirow{3}{*}{$p$} \\
\hline & & & & & & & \\
\hline \multirow{2}{*}{$\begin{array}{l}\text { Smoking } \\
\text { status }\end{array}$} & Smokers & $231.43 \pm 205.22$ & & $11.45 \pm 8.30$ & \multirow[b]{2}{*}{ NS } & $100.25 \pm 1.36$ & \\
\hline & $\begin{array}{l}(n=113) \\
\text { Non-smokers } \\
(n=143)\end{array}$ & $73.22 \pm 73.71$ & $<10^{-7}$ & $12.99 \pm 10.51$ & & $99.60 \pm 0.91$ & $5 \times 10^{-4}$ \\
\hline Gender & $\begin{array}{l}\text { Men }(n=102) \\
\text { Women }(n=11)\end{array}$ & $\begin{array}{l}222.88 \pm 195.76 \\
310.67 \pm 277.40\end{array}$ & 0.03 & $\begin{array}{l}11.16 \pm 8.38 \\
14.09 \pm 7.32\end{array}$ & NS & $\begin{array}{l}100.15 \pm 1.39 \\
100.70 \pm 0.34\end{array}$ & $10^{-4}$ \\
\hline $\begin{array}{l}\text { Alcoholic } \\
\text { beverage }\end{array}$ & $\begin{array}{l}\text { Yes }(n=34) \\
\text { No }(n=79)\end{array}$ & $\begin{array}{l}222.19 \pm 191.35 \\
235.40 \pm 211.96\end{array}$ & NS & $\begin{array}{r}9.95 \pm 6.45 \\
12.09 \pm 8.94\end{array}$ & NS & $\begin{aligned} 100.37 & \pm 1.5 \\
99.80 & \pm 0.51\end{aligned}$ & 0.03 \\
\hline
\end{tabular}

NS: No significant. 
Table 2. Correlation between Urinary Cotinine, Urinary and Plasma $\mathrm{SCN}^{-}$Levels and Consumption Duration, cigarettes smoked/day and BMI

\begin{tabular}{llcrc}
\hline \hline \multicolumn{1}{c}{ Parameters } & $\begin{array}{c}\text { Urinary cotinine } \\
(\mu \mathrm{g} / \mu \mathrm{mol} \mathrm{Cr})\end{array}$ & $\begin{array}{c}\text { Urinary SCN } \\
(\mathrm{mg} / \mathrm{g} \mathrm{Cr})\end{array}$ & $\begin{array}{c}\text { Plasma SCN }^{-} \\
(\mu \mathrm{mol} / \mathrm{l})\end{array}$ \\
\hline Consumption & {$[1-<5](n=15)$} & $158.61 \pm 230.05$ & $11.26 \pm 8.19$ & $100.19 \pm 1.48^{*}$ \\
duration & {$[5-<15](n=50)$} & $222.23 \pm 187.40$ & $12.23 \pm 10.42$ & $100.21 \pm 1.57$ \\
(years) & {$[15-20](n=22)$} & $252.34 \pm 195.97^{*}$ & $11.70 \pm 5.80$ & $100.42 \pm 0.15^{*}$ \\
& $>20(n=26)$ & $272.88 \pm 228.75^{*}$ & $9.83 \pm 5.10$ & $100.43 \pm 1.07^{*}$ \\
Cigarettes & {$[5-10](n=21)$} & $133.89 \pm 149.04^{*}$ & $13.47 \pm 10.96$ & $99.47 \pm 0.41^{*}$ \\
$($ smoked/day) & {$[11-20](n=61)$} & $217.07 \pm 204.90$ & $10.20 \pm 6.70$ & $100.31 \pm 1.48$ \\
& {$[21-30](n=15)$} & $309.09 \pm 194.44^{*}$ & $13.41 \pm 11.94$ & $100.89 \pm 1.55^{*}$ \\
& $>30(n=16)$ & $341.38 \pm 220.29^{*}$ & $11.68 \pm 5.08$ & $102.07 \pm 2.95^{*}$ \\
BMI $\left(\mathrm{kg} / \mathrm{m}^{2}\right)$ & $<25$ & $251.86 \pm 216.65$ & $10.92 \pm 7.37$ & $100.24 \pm 1.44$ \\
& {$[25-<27]$} & $196.00 \pm 111.96$ & $9.05 \pm 6.79$ & $100.00 \pm 0.69$ \\
& {$[27-30]$} & $135.64 \pm 137.59$ & $10.21 \pm 5.46$ & $100.62 \pm 1.63$ \\
& $>30$ & $50.26 \pm 65.46$ & $10.40 \pm 3.97$ & $100.93 \pm 2.04$ \\
\hline
\end{tabular}

${ }^{*} p<0.05$.

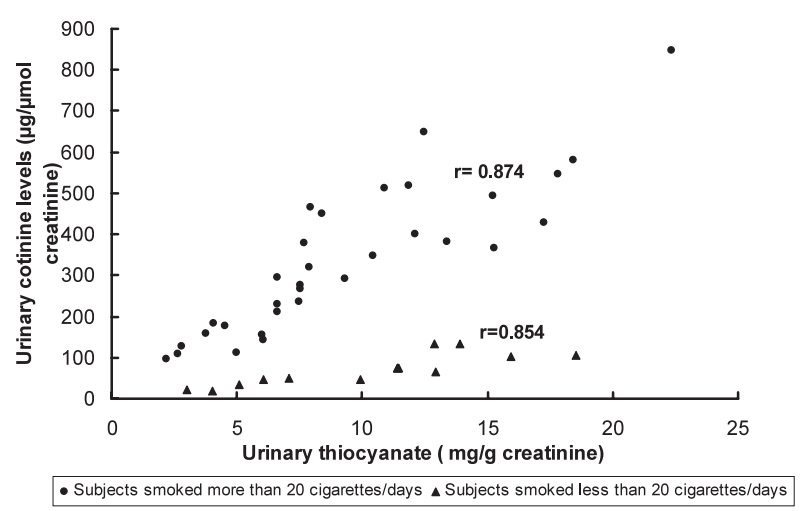

Fig. 1. Correlation between Urinary Cotinine and Urinary $\mathrm{SCN}^{-}$

$\mathrm{SCN}^{-}$levels were significantly higher in women $(p=0.03)$ than in men. For urinary $\mathrm{SCN}^{-}$levels there was no significant difference between the two genders.

There was no significant difference in the urinary cotinine and $\mathrm{SCN}^{-}$levels between the alcoholconsuming and nonconsuming groups, but there was in the plasma $\mathrm{SCN}^{-}$level $(p=0.03)$.

At all ages, $\mathrm{SCN}^{-}$and urinary cotinine distributions in smokers were shifted upward compared with nonsmokers, but when the distributions overlapped no major differences were seen according to age.

Table 2 illustrates the significant correlation between urinary cotinine level and duration of smoking $\left(\mathrm{F}_{3-109}=3.43 ; p=0.019 ; r=0.9961\right)$. However, for plasma and urinary $\mathrm{SCN}^{-}$levels, we found no significant difference between groups, but we noted a slight difference for the plasma $\mathrm{SCN}^{-}$concentration. We found an important correlation between

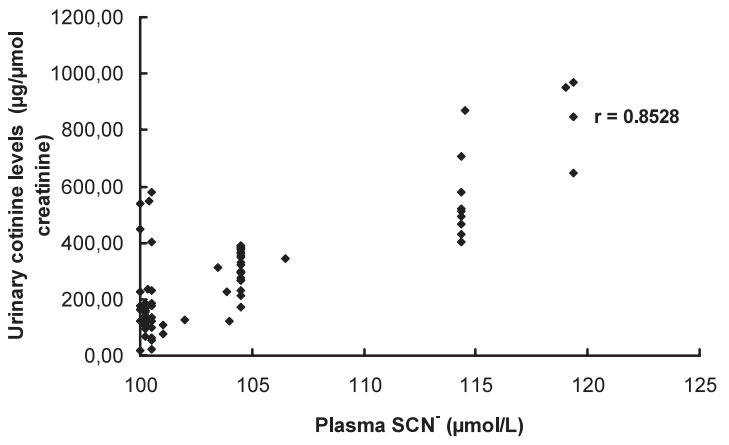

Fig. 2. Correlation between Urinary Cotinine and Plasma $\mathrm{SCN}^{-}$Concentrations

the plasma $\mathrm{SCN}^{-}$level $\left(\mathrm{F}_{3-109}=3.71 ; p=0.014\right.$; $r=0.9899)$ and number of cigarettes consumed per day and between the cotinine level and number of cigarettes per day $\left(\mathrm{F}_{3-109}=4.27 ; p=0.007 ; r=\right.$ $0.9899)$, contrary to urinary $\mathrm{SCN}^{-}$. We found a significant negative correlation between BMI and urinary cotinine, but not for the other parameters.

Figures 1 and 2 show that the correlation between urinary cotinine and plasma $\mathrm{SCN}^{-}$levels $(r=$ $0.8528)$ is greater than that between urinary cotinine and urinary $\mathrm{SCN}^{-}(r=0.4737)$, but only when individuals smoked more than 20 cigarettes per day was the correlation significant $(r=0.8251)$.

\section{DISCUSSION}

In this study we have expressed the results for tobacco markers as concentrations $(\mu \mathrm{mol} / \mathrm{l})$, and for urinary tobacco markers as ratios to creatinine excretion. Thompson et al. demonstrated that the ad- 
justment of urinary cotinine to creatinine improves the relationship between urinary and serum cotinine in smokers. ${ }^{3)}$ Because urinary creatinine excretion is related to muscle in humans, it is at least theoretically relatively stable in healthy individuals. ${ }^{4)}$ However, the value can be influenced by diet or physical activity and urine flow. ${ }^{1)}$

This study found a large overlap in the concentration of urinary cotinine for smokers and nonsmokers of 231.43 and $73.22 \mu \mathrm{g} / \mu \mathrm{mol}$ creatinine, respectively. There was a significant difference between these two groups $\left(p<10^{-7}\right)$. Although cotinine is influenced by diet and industrial pollution, our data show that it remains a reliable indicator of smoking status. ${ }^{1)}$

Cotinine in body fluids is the most frequently used biomarker of tobacco smoke exposure. ${ }^{5,6}$ Cotinine has been shown to be the most specific and most sensitive marker; however, the urinary cotinine concentration is regarded as the best biomarker available for detection of exposure to tobacco smoke and for discriminating active smokers from nonsmokers. A mean of $70-80 \%$ of nicotine is converted to cotinine, which has a half-life of about $17 \mathrm{hr}^{5}{ }^{5}$ We noted that the urinary cotinine level was significantly correlated with the number of cigarettes smoked per day and was significantly positively correlated with the self-reported number of cigarettes smoked per day $\left(\mathrm{F}_{3-109}=\right.$ 4.27; $p=0.007 ; r=0.9899)$. However, cotinine is no longer considered the major metabolite of nicotine; which probably explains why the urinary cotinine level is only roughly related to daily cigarette consumption, because the correlation of urinary cotinine with the number of cigarettes smoked per day is related to that observed in serum or plasma specimens. ${ }^{1)}$ Among smokers, there is a relatively high degree of interindividual variability in cotinine levels among those claiming to smoke the same number of cigarettes per day, although light smokers $(<20 /$ day $)$ had considerably lower mean levels than heavier smokers ( $>20 /$ day). Other investigators found similar levels of variability, which presumably represent differences in individual metabolism and inhalation patterns or the amount of each cigarette smoked. ${ }^{1)}$

In this study, we found a significant correlation between mean urinary cotinine levels and duration of consumption. This correlation can be explained by the long halflife of cotinine, which is eliminated from the body after a few days and is mainly excreted in the urine. Moreover, smoking induces changes in nicotine disposition: the rate of cotinine disappearance from the urine is significantly slower in smokers than in nonsmokers. ${ }^{1)}$ Therefore the determination of this marker in urine is a good alternative to discriminate smokers from nonsmokers.

There was a significant difference in mean urinary cotinine levels between male and female smokers (222.88vs. $310.67 \mu \mathrm{g} / \mu \mathrm{mol}$ creatinine). This difference can be explained by traditional tobacco use and diet, because woman tend to eat more vegetables than men, and plant sources others than tobacco have been reported to contain varying levels of nicotine. Many such plants are common dietary constituents, for example, eggplant, potato, and tomato, and might contribute to urinary cotinine levels to a different degrees for former and never smokers. However, Benowitz estimated the amount in several nicotine-containing vegetables which would be required daily to yield a urinary cotinine level of $\left.1 \mu \mathrm{g} / \mathrm{l}^{5}\right)$

Urinary cotinine levels were significantly correlated with W; the correlation of cotinine levels with $\mathrm{W}$ in smokers was not surprising, because nicotine intake suppresses body $\mathrm{W}$ in a dose-related fashion. ${ }^{7)}$ The BMI for smokers was significantly lower than for nonsmokers, although they consumed more calories than nonsmokers. This finding supports the hypothesis that smokers have a higher metabolic rate than nonsmokers, as previously described. ${ }^{7)}$ However, that group of patients also had lower mean age and included more men than other groups. Moreover, their alcohol intake might adversely impact the absorption of nutrients or participate in the nonenergy-producing microsomal system. ${ }^{1)}$

$\mathrm{SCN}^{-}$has been extensively studied in plasma and urine. Our results show that only $\mathrm{SCN}^{-}$measured in plasma discriminates smokers from nonsmokers, and agree with those obtained by Jarvis et al., ${ }^{8)}$ who reported that plasma $\mathrm{SCN}^{-}$has been used as an objective index of smoking because of its long halflife and ability to distinguish smoking from nonsmoking populations.

In this study we found a strong correlation between plasma $\mathrm{SCN}^{-}$levels and number of cigarettes per day $\left(\mathrm{F}_{3-109}=3.71 ; p=0.014 ; r=0.9899\right)$, unlike the urinary $\mathrm{SCN}^{-}$correlation in the study of William. ${ }^{9)}$ The association with depth of inhalation (smoking vs. nonsmoking) was dominant among the relationships considered.

Our results showed that the mean plasma $\mathrm{SCN}^{-}$ level in nonsmokers was $100.15 \mu \mathrm{mol} / \mathrm{l}$ for men and $100.7 \mu \mathrm{mol} / \mathrm{l}$ for women. One of the few studies 
to report findings in men and women separately was that of Tenovuo and Makinen. ${ }^{10)}$ These authors found different $\mathrm{SCN}^{-}$levels in the two genders. Although some of the difference between genders may be due to the amounts smoked or other smoking habits, nonetheless, the significant difference between male and female nonsmokers indicates that cyanide may not be metabolized in the same way by men and women. Investigators should use caution in extrapolating findings such as reported in this study from women to men. Nonsmokers tend to eat more vegetables than smokers which may increase the plasma $\mathrm{SCN}^{-}$level. ${ }^{11)}$ In the U.S.A., where high consumption of vegetables is still uncommon, ${ }^{12)}$ the influence on $\mathrm{SCN}^{-}$levels is probably slight The difference between the $\mathrm{SCN}^{-}$levels in women and men smoking the same number of cigarettes can be explained by the gender difference in the distribution volume of $\mathrm{SCN}^{-}$. Among nonsmokers, the mean level of serum $\mathrm{SCN}^{-}$was the same whether they had been indirectly exposed to tobacco smoke or not. The mean plasma $\mathrm{SCN}^{-}$levels were up to $10 \mu \mathrm{mol} / \mathrm{l}$ higher in the last half of the year than in the first half. This can be explained by seasonal variations in the content of $\mathrm{SCN}^{-}$in the diet. The range of the individual $\mathrm{SCN}^{-}$level was great in both nonsmokers and smokers, resulting in a large overlap. In conclusion, a number of characteristics of $\mathrm{SCN}^{-}$make it a useful index of smoking exposure. First, numerous authors have reported that $\mathrm{SCN}^{-}$levels discriminate smokers from nonsmokers. ${ }^{13)}$ Second, $\mathrm{SCN}^{-}$levels have shown doseresponse relationships with the rate of cigarette consumption. ${ }^{14,15)}$ Third, the $\mathrm{SCN}^{-}$level has been reported to be sensitive to changes in smoking patterns such as the brand of cigarettes smoked.

In this study, we established a strong correlation between urinary cotinine and plasma $\mathrm{SCN}^{-}$levels $(r$ $=0.8528)$. This association is greater than the correlation among urinary cotinine and urinary $\mathrm{SCN}^{-}$, and plasma $\mathrm{SCN}^{-}$and urinary $\mathrm{SCN}^{-}$. An important correlation $(r=0.825)$ was noted between urinary cotinine and urinary $\mathrm{SCN}^{-}$only for individuals who smoked more than 20 cigarettes per day, indicating that either the type of smoker in regard to behavior dependence was different or that tolerance and/or altered metabolism occurred. The present results are in line with those of previous studies combining cotinine and $\mathrm{SCN}^{-}$.6)

Half-life varies between 14 and $17 \mathrm{hr}$ for cotinine ${ }^{6)}$ and between 5 and 15 days for $\mathrm{SCN}^{-},{ }^{13)}$ and thus irregular consumption of cigarettes may induce a constant, high level of $\mathrm{SCN}^{-}$but a relatively low level of cotinine between episodes of smoking. Alternatively, cotinine can be high and $\mathrm{SCN}^{-}$low if a usually light smoker smoked a cigarette shortly before the interview. Observations in individuals with nonconcordant levels of cotinine and $\mathrm{SCN}^{-}$support this hypothesis. ${ }^{16)}$

Self-reported, current smoking was found to be poorly sensitive when only cotinine or $\mathrm{SCN}^{-}$was used as biomarker of exposure to tobacco smoke, but highly sensitive when the combination of both biomarkers was used.

In conclusion, cotinine and $\mathrm{SCN}^{-}$concentrations were determined in urine and plasma samples from 256 healthy young persons (133 smokers) and related to their smoking and physiologic characteristics. Urinary cotinine and plasma $\mathrm{SCN}^{-}$ concentration were both significantly higher in selfreported smokers than in nonsmokers and correlated well with the number of cigarettes smoked per day and with the duration of consumption, although the reverse was found for urinary $\mathrm{SCN}^{-}$.

This study confirms the utility of plasma $\mathrm{SCN}^{-}$, and urinary cotinine as an index of smoking rate and demonstrates the role of secondary variables in accounting for the chemical in biological fluids.

We conclude that the combination of cotinine and $\mathrm{SCN}^{-}$concentrations was preferable to either alone in classifying smoking status.

Acknowledgements This research was supported by the research team of the Laboratory of Biochemistry-Toxicology, CHU Fattouma Bourguiba, Monastir, Tunisia.

\section{REFERENCES}

1) Brukert, E., Jacob, N., Lamaire, L., Truffert, J., Percheron, F. and Gennes, L. (1992) Relationship between smoking status and serum lipids in a hyperlipidemic population and analysis of possible confounding factors. Clin. Chem., 38, 1698-1705.

2) Densen, P. M., Davidow, B., Bass, H. E. and Jones, E. W. (1967) A chemical test for smoking exposure. Arch. Environ. Health, 14, 865-874.

3) Thompson, S. G., Barlow, R. D., Wald, N. J. and Van Vunnakis, H. (1990) How should urinary cotinine concentration be adjusted for urinary creatinine concentration? Clin. Chim. Acta, 187, 289-296.

4) Heymsfield, S. B., Arteaga, C., McManus, C., Simith, J. and Moffit, S. (1983) Measurement of 
muscle mass in humans: validity of the 24-hour urinary creatinine method. Am. J. Clin Nutr., 37, 478494.

5) Benowitz, N. L. (1996) Cotinine as a biomarker of environmental tobacco smoke exposure. Epidemiol. Rev., 18, 188-200.

6) Etzel, R. A. (1990) A review of the use of saliva cotinine as a marker of tobacco smoke exposure. Prev. Med., 19, 190-197.

7) Grunberg, N. E., Bowen, D. J., Maycock, V. A. and Nespor, S. M. (1985) The importance of sweet taste and caloric content in the effects of nicotine on specific food consumption. Psychopharmacology Biochemistry Behaviours, 23, 851-854.

8) Jarvis, M. J., Tunstall-Pedoe, H., Feyerabend, C., Vesey, C. and Salloojee, Y. (1984) Biochemical markers of smoke absorptionand self-reported exposure to passive smoking. J. Epidemiol. Community Health, 38, 335-339.

9) William, C. B., Martha, K. and Graham, M. W. (1974) Automated Method for determining serum thiocyanate, to distinguish smokers from nonsmokers. Clin. Chem., 20, 1344-1348.

10) Tenovuo, J. and Makinen, K. (1976) Concentration of thiocyanate and ionizable iodine in saliva of smokers and non-smokers. J. Dent. Res., 55,
661-663.

11) Subar, A. F., Harlan, L. C. and Mattson, M. E. (1990) Food and nutrient intake differences between smokers and non-smokers in the U.S. Am. J. Public Health, 80, 1323-1329.

12) Patterson, B. H., Block, G., Rosenberger, W. F., Pee, D. and Kahle, L. L. (1990) Fruit and vegetables in the American diet: Data from the NHANES II survey. Am. J. Public Health, 80, 1443-1449.

13) Donald, M. P., John, E. M., Arthur, S. H. and Norman, S. (1981) The reliability of thiocyanate measurment of smoking exposure. Addict. Behav., 6, 99-105.

14) Butts, W. C., Kuehneman, M. and Widdowson, G. M. (1974) Automated method for determining serum thiocyanate to distinguish smokers from nonsmokers. Clin. Chem., 20, 1344-1348.

15) Vogt, T. M. (1977) Smoking behavioural factors as predictors of risk. Research Smoking Behaviours, I, 58-78.

16) Morabia, A., Bernstein, M. S., Curtin, F. and Berode, M. (2001) Validation of Self-Reported Smoking Status by Simultaneous Measurement of carbon monoxide and salivary thiocyanates. Prev. Med., 32, 82-88. 